\title{
Theranostics: Fusion of Therapeutics and Diagnostics
}

\author{
Zheng-Rong Lu
}

Received: 24 February 2014 / Accepted: 24 February 2014 / Published online: 13 March 2014

(C) Springer Science+Business Media New York 2014

Advancements in biomedical imaging have made a revolutionary impact on human healthcare since Dr. Wilhelm Conrad Rontgen imaged his wife's hand on an X-ray machine in 1895. Biomedical imaging allows non-invasive visualization of the anatomic structures of human internal organs and tissues, which provides factual information about human diseases for accurate diagnosis. Recent progress in imaging technologies further extends diagnostic imaging from anatomic imaging to molecular imaging, and qualitative to quantitative visualization of the biological processes of human diseases at the molecular level. The clinical application of modern biomedical imaging has been expanded from traditional diagnostic imaging to almost every aspect of medical practice, including disease diagnosis and prognosis, image guidance in therapeutic interventions and noninvasive assessment of therapeutic efficacy. Molecular imaging also enables non-invasive determination of the pharmacokinetics and pharmacodynamics of therapeutics. Biomedical imaging has been used along with therapy to tailor personalized therapeutic strategies to treat life-threatening diseases and to achieve the best possible therapeutic outcome.

Theranostics is the fusion of therapeutics and diagnostics, the combination of diagnosis and therapy to design individualized pharmacotherapy. Theranostics examines a diverse set of genomic, proteomic and metabolic biomarkers and surrogate biomarkers, e.g. imaging biomarkers. Theranostics identifies suitable molecular targets to treat with the best possible therapies and eliminates ineffective therapies. Theranostics determines the pharmacokinetics and pharmacodynamics of a drug and tailors the best possible therapeutic and dosing strategies for individual patients. The theranostic strategy has been used to design new therapeutics in combination with molecular imaging. There has been significant progress in the design and development of theranostics. This special issue exemplifies some directions of the research in theranostics, especially using biocompatible materials in the design of new theranostics.

\section{Z.-R. Lu $(\bowtie)$}

Department of Biomedical Engineering, Case Western Reserve

University, Cleveland, Ohio 44106, USA

e-mail: zxII25@case.edu
Three review articles in the issue summarize the recent development of the theranostics based on biocompatible polymers, paramagnetic nanoparticles and other platforms. These nanosized materials are modified or functionalized to accommodate the agents of different functions, including targeting, imaging and therapy, for preparing theranostics. Biocompatible polymers have been extensively investigated for drug delivery, and some of the delivery systems have been used in clinical practice. They are versatile platforms for design and preparation of theranostics and have shown some advantageous features for imaging and treating human diseases (Wang, et al.). The incorporation of imaging agents into various drug delivery systems allows non-invasive determination of the pharmacokinetics and pharmacodynamics of the drug delivery systems, which provide valuable information for further optimization and development of the theranostics for imaging and treating life-threatening diseases, including cancer and cardiovascular diseases (Ai, et al.). Paramagnetic nanoparticles were initially used as contrast agents for magnetic resonance imaging (MRI). Surface modification of these nanoparticles introduces various functions for the nanoparticles to be used for both gene delivery and MR imaging. The combination based on the nanoparticles allows non-invasive monitoring of in vivo gene delivery with MRI and delivery of therapeutic genes (Xing, et al.).

Imaging agents are a critical component of theranostics and can facilitate accurate diagnosis of human diseases and therapeutic efficacy assessment. There is an unmet clinical need for safe and effective imaging agents for early accurate diagnosis of cancer and cardiovascular diseases. Sun et al. have modified gold nanoparticles with glycol chitosan to prepare a tumor targeting contrast agent for cancer imaging with X-ray computed tomography (CT). Gold nanoparticles have a high X-ray attenuation efficiency and are efficient CT contrast agents. The surface modification promotes the uptake of the nanoparticles in tumor cells to enhance tumor contrast in CT. El-Dakdouki et al. have modified paramagnetic nanoparticles to target the cell surface adhesion molecule CD44 in atherosclerotic plaques. The agent specifically bound to the biomarker model and produced significant plaque enhancement in a rabbit in $\mathrm{T} 2{ }^{*}$ weighted MR images. 
Intracellular delivery of theranostics is a critical step for diagnosis and treatment of life-threatening human diseases. Lin et al. have identified scavenger receptor class B type I (SR$\mathrm{BI})$, a receptor for high density lipoprotein (HDL), as a molecular target for cytosolic delivery of theranostics using HDLlike nanoparticles for cancer diagnosis and therapy. The mechanism of intracellular drug delivery via SR-BI is elucidated in this work by using fluorescence imaging. The group has discovered that SR-BI mediates cytosolic drug delivery mainly through a lipid raft/caveolae-like pathway. Understanding the mechanism is valuable to utilize the pathway to design better cytosolic delivery systems for theranostics into target cells.

The incorporation of imaging agents into drug formulations allows non-invasive determination of their pharmacokinetics and pharmacodynamics in both drug development and clinical practice. Hollis et al. have developed hybrid paclitaxol nanoparticles containing a bioactivatable fluorescence probe (MMPSencse 750 FAST) and a near infrared probe (Flamma Fluor FPR-648) to track in vivo delivery of the anticancer drug and to assess therapeutic efficacy using fluorescence imaging in a breast cancer mouse model. The hybrid nanocrystals act as theranostics for monitoring tumor status and pharmacokinetics of the drug nanoparticles. The MMPSense dye is activated by matrix metalloproteinase (MMP) in solid tumors and is used to monitor tumor progression and therapeutic efficacy. FPR-648 is used for monitoring the drug delivery process and pharmacokinetics. Since the probes have different emission wavelengths, they can be imaged simultaneously in an animal model. The hybrid theranostics provide non-invasive determination of real time pharmacokinetics, in vivo drug release and pharmacodynamics of the nanocrystals.

Pubudu et al. have developed an interesting nanotrain construct comprising three paramagnetic iron oxide nanoparticles and one liposome containing doxorubicin for radiofrequency-triggered drug release to treat aggressive breast cancer. The iron oxide nanoparticles, nanosized MRI contrast agents, are used to trigger drug release from the liposomes caused by the oscillation of the iron oxide nanoparticle chain in response to applied radiofrequency. The oscillation of the paramagnetic tail in the train mechanically opens the "door" in the liposomal "cargo container" to unload the drug in the tumor when the train receives the "unloading radiofrequency signal". The novel nanotrain construct results in more significant antitumor efficacy in a tumor model after radiofrequency activation than the free and the unactivated nanotrain.

Perera et al. have prepared Pluronic containing nanosized bubbles as theranostics for hyperthermia cancer therapy. Pluronic stabilizes the nanobubbles and possesses the ability of sensitizing multidrug resistant cancer cells to enhance anticancer therapeutic efficacy. The nanobubbles accumulate in tumor tissue and can be visualized by ultrasound imaging. Ultrasound is then used to enhance cellular uptake of Pluroic to sensitize cancer cells and to localize the tumor to guide phototherapy. The nanobubbles with ultrasound modulation significantly enhance the antitumor efficacy of thermotherapy than thermotherapy alone.

Theranostics can also be used to detect and localize tumors to guide precise tumor resection in surgery. Tan et al. have developed a targeted dual imaging agent containing an MRI contrast agent and fluorescence probe for non-invasively detecting the tumor with MRI, pre-surgical planning and visualizing the tumor margin with fluorescence imaging to guide the surgery. The reported targeted dual imaging agent specifically binds to a tumor biomarker in the extracellular matrix of orthotopic prostate cancer in a mouse model. The agent produced significant contrast enhancement of the tumor in the MR images and strong fluorescence signal in the tumor tissue.

Theranostics involves multiple disciplines, including engineering, chemistry, biology, pharmaceutical science and medicine. This multidisciplinary approach has a great potential to revolutionize human healthcare and to provide better patient care. Safe and effective diagnostics and therapeutics are the central piece of theranostics. They play a critical role in the characterization of various biomarkers and effective treatment of human diseases accordingly. Despite the progress in the development of novel theranostics, tremendous hurdles need to be overcome for translational development of nanosized theranostics for clinical applications. Nevertheless, theranostics opens a brand new avenue for tailoring personalized patient care and gives a hope for the cure of lifethreatening human diseases.

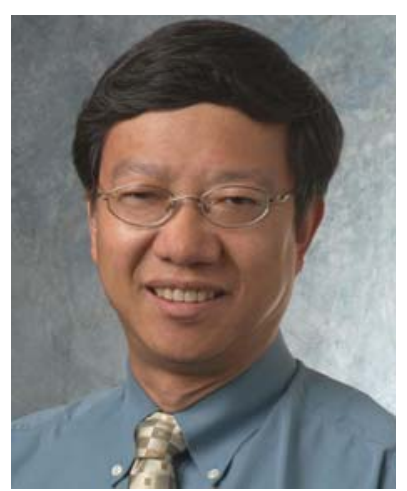

Dr. Zheng-Rong Lu is M. Frank and Margaret Domiter Rudy Professor of Biomedical Engineering at the Department of Biomedical Engineering, Case School of Engineering, Case Western Reserve University. Dr. Lu received his B.S. and M.S. in Chemistry from Lanzhou University, and Ph.D. in Chemistry from Lanzhou Institute of Chemical Physics, Chinese Academy of Sciences at Lanzhou, China. Dr. Lu was an Associate Professor of Chemistry in 1992 and Professor of Chemistry in 1994 at Wuhan University in China. In 2002, Dr. Lu was appointed to Assistant Professor in the Department of Pharmaceutics and Pharmaceutical Chemistry at the University of Utah. Dr. Lu was promoted to tenured Associate Professor in 2006. Dr. Lu's research involves molecular imaging, MRI contrast agents, image-guided photodynamic therapy, non-invasive methods for cancer treatment 
assessment, drug delivery systems for retinal diseases and multifunctional delivery systems for nucleic acids. He has over 120 peerreviewed scientific publications, five book chapters and 70 abstracts and four US patents. He is a Principal Investigator of major grants from the NIH. Dr. Lu serves on the scientific advisory board of Pharmaceutical Research, Molecular Pharmaceutics, ISRN Bioma- terials and American Journal of Nuclear Medicine and Molecular Imaging. Dr. Lu served as a member of the NIH Clinical Molecular Imaging and Probe Development Study Section from 2009 to 2013. He was a co-convener of 2013 Symposium of Contrast Media Research. Dr. Lu is elected to the College of Fellows the American Institute for Medical and Biological Engineering (AIMBE). 